Article

\title{
Discretely Tunable Multiwavelength Visible Laser Based on Cascaded Frequency Conversion Processes
}

\author{
Xinlin Lv ${ }^{1,2,+}{ }^{\oplus}$, Junchi Chen ${ }^{1,+}$, Yujie Peng ${ }^{1, *}$, Zhiyuan Huang ${ }^{1,2}$, Yingbin Long ${ }^{1}$, \\ Xinliang Wang ${ }^{1}$, Guanting Liu $^{1}$ and Yuxin Leng ${ }^{1, *}$ \\ 1 State Key Laboratory of High Field Laser Physics and CAS Center for Excellence in Ultra-intense Laser \\ Science, Shanghai Institute of Optics and Fine Mechanics (SIOM), Chinese Academy of Sciences (CAS), \\ Shanghai 201800, China; lvxinlin@siom.ac.cn (X.L.); chenjunchi0226@siom.ac.cn (J.C.); \\ huangzhiyuan@siom.ac.cn (Z.H.); longyingbin@siom.ac.cn (Y.L.); wxl@siom.ac.cn (X.W.); \\ liuguanting@siom.ac.cn (G.L.) \\ 2 Center of Materials Science and Optoelectronics Engineering, University of Chinese Academy of Sciences, \\ Beijing 100049, China \\ * Correspondence: yjpeng@siom.ac.cn (Y.P.); lengyuxin@mail.siom.ac.cn (Y.L.); \\ Tel.: +86-021-6991-8753 (Y.P.); +86-021-6991-8436 (Y.L.) \\ + These authors contributed equally to this work.
}

Received: 12 November 2020; Accepted: 30 November 2020; Published: 1 December 2020

check for updates

\begin{abstract}
We demonstrate a discretely tunable multiwavelength visible laser through second harmonic generation (SHG) and sum frequency generation (SFG) of multiorder Stokes lasers generated from an external Raman laser oscillator. The Raman laser oscillator, driven by a $1064 \mathrm{~nm}$ laser with an energy of $120 \mathrm{~mJ}$, is based on a cascade of $\mathrm{Ba}\left(\mathrm{NO}_{3}\right)_{2}$ and two axial orthogonal $\mathrm{KGd}\left(\mathrm{WO}_{4}\right)_{2}$ crystals. Through adjusting the angle of the SHG/SFG crystal, we obtain 16 visible wavelengths with a wide range from $579.5-658.4 \mathrm{~nm}$. In addition, we investigate the output energy and conversion efficiency of the resulting laser with various phase-matching angles. We show that the maximum energy of the visible laser is $8.87 \mathrm{~mJ}$ with five wavelengths, and the corresponding total conversion efficiency is $7.4 \%$. These experimental results demonstrate a practical and effective method of generating a discretely tunable multiwavelength visible laser.
\end{abstract}

Keywords: visible laser; all-solid-state laser; stimulated Raman scattering; multiwavelength generation

\section{Introduction}

The development of visible lasers with multiwavelength emission simultaneously is popular due to their wide applications in optical storage, airborne LiDAR, laser display, pump probe, multispectral analysis, and terahertz generation [1-9]. Currently, there are several techniques to produce multiwavelength lasers. Diversified laser mediums and nonlinear effects make it possible to produce multiwavelengths at the same time. Take the stimulated Raman scattering (SRS) process as an example; the output spectra of the Stokes lasers can be manipulated through the design of the mirror transmission parameters [10-13]. Moreover, most of the output lasers reside in the near-infrared range and can be converted into visible lasers through second-order nonlinear processes, including second harmonic generation (SHG) and sum frequency generation (SFG) $[14,15]$. Second-order nonlinear optical effects, such as optical parametric oscillator (OPO) [16], and third-order nonlinear optical effects, such as the SRS and stimulated Brillouin scattering (SBS), can also be used to realize frequency conversion, effectively enriching the visible laser spectrum.

SRS has been applied to the frequency conversion of gas, liquids, and solids-in particular, solid-state Raman media with characteristics of high ion density, large gain coefficient, wide frequency 
shift range, and large thermal conduction coefficient [17]. A multiorder Stokes laser can be achieved by SRS process when the pumping laser intensity is high enough $[18,19]$. Previously, numerous solid-state Raman mediums have been developed, including CVD diamond [20-22], $\mathrm{YVO}_{4}$ crystal [23,24], $\mathrm{Ba}\left(\mathrm{NO}_{3}\right)_{2}$ crystal [25-27], and $\mathrm{KGd}\left(\mathrm{WO}_{4}\right)_{2}(\mathrm{KGW})$ crystal [28]. Specifically, $\mathrm{Ba}\left(\mathrm{NO}_{3}\right)_{2}$ crystal is a prevailing solid-state Raman gain media due to its high Raman gain coefficient of approximately $11 \mathrm{~cm} / \mathrm{GW}$ at $1064 \mathrm{~nm}$ wavelength and narrow Raman line-width. Additionally, KGW crystal is gaining popularity as a Raman gain medium characterizing due to its relatively high thermal conductivity, a wide optical transmission window $(0.35-5.5 \mathrm{um})$, and high damage threshold of $>10 \mathrm{GW} / \mathrm{cm}^{2}$. Moreover, KGW crystal has a monoclinic lattice structure, meaning it has anisotropic optical properties. Consequently, two different Raman shifts of $768 \mathrm{~cm}^{-1}$ (p [gg]p) and $901 \mathrm{~cm}^{-1}$ (p[mm]p) by KGW crystals make it possible to generate two different Stokes outputs, as determined by the polarization of pumping laser. In addition, compared with SRS generation, SRS oscillators have the obvious advantage of higher efficiency.

Furthermore, the combination of SRS with second-order nonlinear process, especially frequency up-conversion processes such as SHG and SFG, has been demonstrated to be an effective technique to achieve the multiwavelength laser output $[29,30]$. Spence et al. conducted a series of experiments, successfully obtaining multiwavelength visible lasers based on the KGW crystals [18,29,31,32]. When $532 \mathrm{~nm}$ lasers were selected as the pumping source for the orthogonal placed KGW crystal, more than eight wavelengths from yellow to red spectral regions, were generated by changing the KGW crystal orientation and output coupler.

In this work, we demonstrate a cascaded infrared Raman laser system combined with the following SHG and SFG technique. Through adjusting the angle of the SHG/SFG crystal, the laser system can emit 16 visible wavelengths with a broad spectral range between 579.5 and $658.4 \mathrm{~nm}$. The system consists of a Raman oscillator and a following potassium dihydrogen phosphate (KDP) based SHG and SFG parts. Gain mediums that are cascaded in the Raman oscillator are one $\mathrm{Ba}\left(\mathrm{NO}_{3}\right)_{2}$ and two axis-orthogonal KGWs crystals, pumped by a nanosecond $1064 \mathrm{~nm}$ laser. Seven Stokes lasers with total energy of $44 \mathrm{~mJ}$ are obtained under the pumping energy of $120 \mathrm{~mJ}$, yielding a Raman conversion efficiency of $36.7 \%$. Subsequently, the multiple Stokes lasers are injected into a KDP crystal for frequency up-conversion into the visible range. Finally, up to 11 visible wavelengths can be simultaneously obtained and are discretely tunable by changing the phase-matching angle of KDP crystal. With maximum output energy of $8.87 \mathrm{~mJ}$, the five visible-wavelength lasers obtain with a SHG/SFG efficiency of $20.1 \%$ (total efficiency of $7.4 \%$ ). These results demonstrate that cascading multiple crystal Raman with second order frequency mixing can expand the wavelength-lines, as desired by their many applications.

\section{Experimental Setup}

A schematic of the discretely tunable multiwavelength visible laser based on cascaded SRS and SHG/SFG is presented in Figure 1. The whole system contains a $1064 \mathrm{~nm}$ pumping laser, an optical isolator, an external Raman laser oscillator, a nonlinear conversion part, and a prism for color separation. To start, a $Q$ switched flash-lamp pumped $1064 \mathrm{~nm}$ laser is utilized, delivering a maximum energy of $400 \mathrm{~mJ}$ with a pulse width of $7.7 \mathrm{~ns}$ at a repetition rate of $10 \mathrm{~Hz}$. For energy adjustment and prevention of residual return laser, a half wave plate (HWP), a polarization beam splitter (PBS), and a Faraday rotator (FR) are placed directly after the $1064 \mathrm{~nm}$ laser. Another HWP follows the FR and is used to change the polarization of the pumping laser. Next, the laser beam, with p-polarization, is focused by a plane-convex lens L1 $(\mathrm{f}=400 \mathrm{~mm})$ and then injected into the Raman oscillator. 


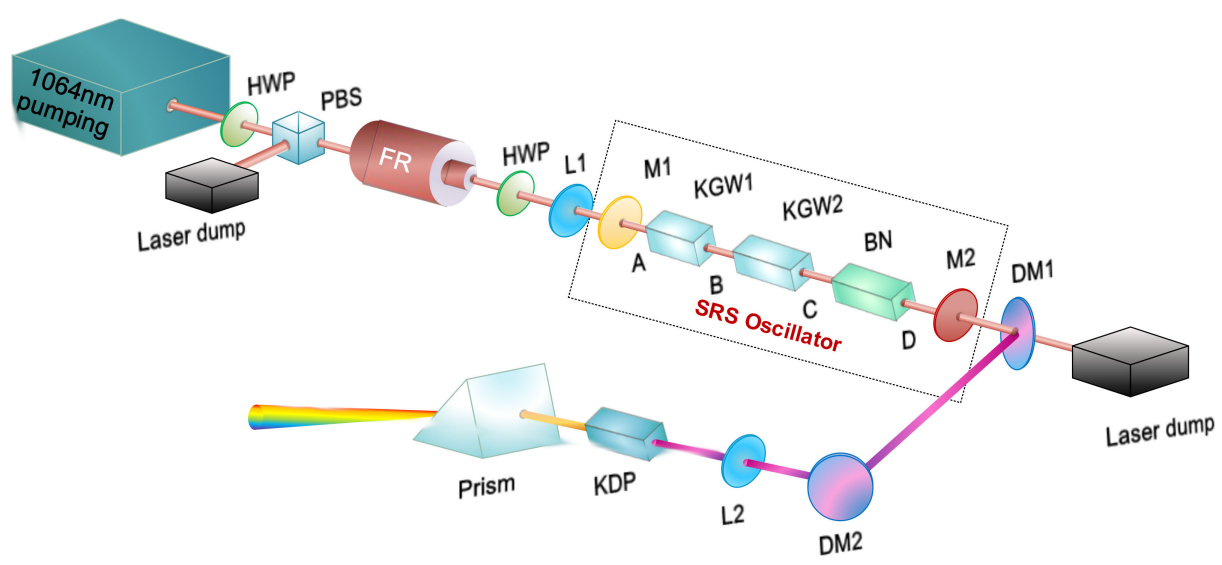

Figure 1. Schematic of the discretely tunable multiwavelength visible laser based on second harmonic generation (SHG), sum frequency generation (SFG) and cascaded stimulated Raman scattering (SRS) system. M1 and M2 form the SRS cavity. HWP: half wave plate; PBS: polarization beam splitter; FR: Faraday rotator; L: lens, M: mirror; $\mathrm{BN}: \mathrm{Ba}\left(\mathrm{NO}_{3}\right)_{2}$; DM: dichroic mirror.

The cavity of the external Raman oscillator is composed of a high reflection mirror (M1) and a partial reflection mirror (M2). M1 is coated with a high reflection film from 1150 to $1400 \mathrm{~nm}$ and antireflection at $1064 \mathrm{~nm}$. M2 is a partial reflection mirror with transmittance of $80 \%$ from 1000 to $1450 \mathrm{~nm}$ and high transmission at $1064 \mathrm{~nm}$. Meanwhile, two axis-orthogonal KGW and one $\mathrm{Ba}\left(\mathrm{NO}_{3}\right)_{2}$ crystals are placed in the Raman oscillator in sequence, as shown in Figure 1. The KGW1 crystal is cut along the b-axis with dimensions of $10 \times 10 \times 50 \mathrm{~mm}$ without coating and is placed after M1 with the $\mathrm{N}_{\mathrm{m}}$ optical axis parallel to the pumping polarization $\left(E \| \mathrm{N}_{\mathrm{m}}\right)$. The KGW2 crystal is cut along the b-axis with dimensions of $10 \times 10 \times 70 \mathrm{~mm}$ without coating and is placed after the KGW1 with the $\mathrm{N}_{\mathrm{g}}$ optical axis parallel to the pumping polarization $\left(\mathrm{E} \mid \mathrm{N}_{\mathrm{g}}\right)$. The $\mathrm{Ba}\left(\mathrm{NO}_{3}\right)_{2}$ crystal, with dimensions of $10 \times 10 \times 80 \mathrm{~mm}$ without coating, is placed after KGW2 crystal. Since the $1064 \mathrm{~nm}$ pumping lasers are focused into the oscillator by L1, the beam diameter was measured at four points-A, B, $\mathrm{C}$, and D-which are about 3, 2, 1.25, and $2 \mathrm{~mm}$ respectively. Two dichroic mirrors, DM1 and DM2, are placed after the SRS oscillator to separate the output Stokes lasers from pumping lasers.

After being reflected by DM2, the multiorder Stokes lasers are focused by a plane-convex lens, L2, with a focal length of $100 \mathrm{~mm}$. For type-I SHG and SFG, an uncoated $5 \times 5 \times 30 \mathrm{~mm} \mathrm{KDP}$ crystal is placed at the focus of the laser beams. Moreover, for accurate azimuth adjustment, the KDP crystal is mounted on a rotation stage. As calculations suggest that the phase-matching angle, $\theta$, for several output Stokes laser is around $42^{\circ}$, the crystal is initially cut for the type-I phase matching with cutting angles of $\theta=42^{\circ}$ and $\varphi=45^{\circ}$.

\section{Experimental Results and Discussion}

\subsection{Stokes Lasers Generation}

The calculated wavelengths and measured spectra of the first, second, and third-order Stokes lasers from the external Raman oscillator are shown in Figure 2. The spectra of the output Stokes lasers produced at the pumping energy of $120 \mathrm{~mJ}$ are measured by a near-infrared spectrometer with a $7 \mathrm{~nm}$ wavelength resolution (Ocean optics, NIR2500). For the $\mathrm{Ba}\left(\mathrm{NO}_{3}\right)_{2}$ crystal with a Raman shift of $1047.3 \mathrm{~cm}^{-1}$, the calculated wavelengths of the first, second, and third-order Stokes laser are 1197.7, 1369.5, and $1598.8 \mathrm{~nm}$, respectively. For the KGW1 crystal (corresponding to $E \| \mathrm{N}_{\mathrm{m}}$ ) with Raman shift of $901 \mathrm{~cm}^{-1}$, the calculated wavelengths of the first, second, and third-order Stokes laser are 1177.1, 1316.7, and $1493.9 \mathrm{~nm}$. For the KGW2 crystal (corresponding to E\| $\mathrm{N}_{\mathrm{g}}$ ) with Raman shift of $768 \mathrm{~cm}^{-1}$, the calculated wavelengths of the first, second, and third-order Stokes laser are 1158.9, 1272.1, and $1409.9 \mathrm{~nm}$. By our calculation, the Kerr lens and thermal lens effects of these Raman crystals are negligible at a pumping energy of $120 \mathrm{~mJ}[33,34]$. According to a cascaded SRS model, the threshold 
of SRS for Stokes laser oscillating is determined by pumping depletion, reflectivity of cavity mirrors, Raman gain coefficient, and the length of the Raman medium [35,36]. In addition, the output energy of Stokes lasers is mostly determined by the intensity of pumping laser, length of Raman medium, and the Raman gain coefficient at the pumping wavelength [37,38]. The optical conversion efficiency from pumping lasers to Stokes lasers is related to the parameters of the pumping pulses (i.e., the pulse duration and intensity), the features of the Raman medium (such as the length and Raman gain coefficient), and especially the reflectivity spectral profiles of the resonator mirrors $[36,39]$. Therefore, the output spectra and energy of the Stokes lasers, from the aforementioned external Raman oscillator, mainly depend on the pumping intensity and transmission parameter of the output coupler, M2.

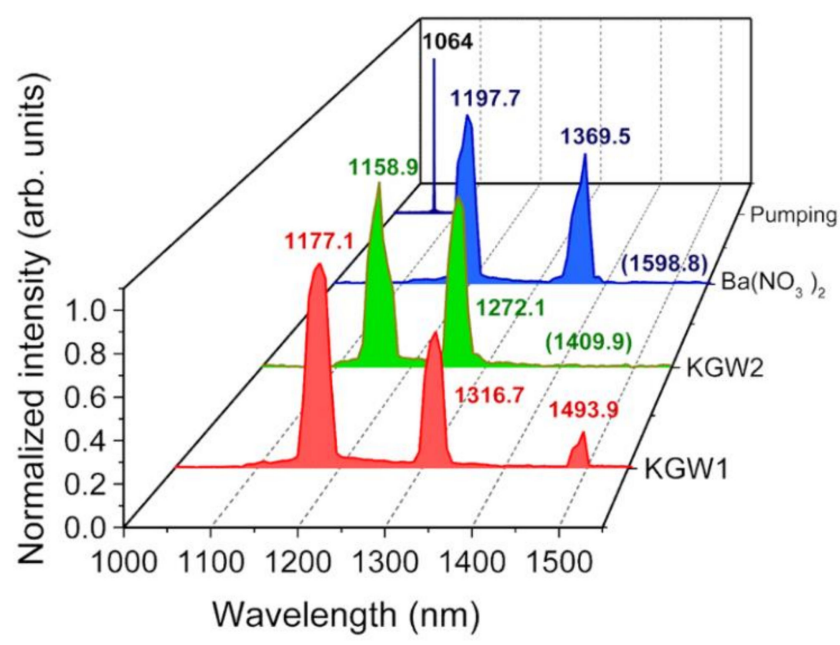

Figure 2. The calculated Stokes wavelengths (numerical value) and the detected spectra (figure) of the Stokes lasers at the pumping energy of $120 \mathrm{~mJ}$. The calculated Stokes wavelengths of 1409.9 and $1598.8 \mathrm{~nm}$ are not detected in the experiments.

At the beginning, the $1064 \mathrm{~nm}$ pumping laser pulses of $20 \mathrm{~mJ}$ are injected into the Raman oscillator, and only the $1197.7 \mathrm{~nm}$ laser appears, since the SRS threshold is related to the Raman gain coefficient, for which $\mathrm{Ba}\left(\mathrm{NO}_{3}\right)_{2}$ crystal is higher than KGW crystal. In addition, the reflectivity coefficient of the two cavity mirrors are almost the same at wavelengths of the three first-order Stokes lasers. As the pumping energy increases to $40 \mathrm{~mJ}$, the first-order Stokes lasers of the three crystals are generated at $1197.7,1177.1$, and $1158.9 \mathrm{~nm}$. Furthermore, when the energy of the pumping laser is increased to $60 \mathrm{~mJ}$, three second-order Stokes lasers are generated at 1316.9, 1272.1, and $1369.5 \mathrm{~nm}$ in addition to the previous first-order Stokes lasers. When the energy of the pumping laser is further increased to $120 \mathrm{~mJ}$, the third-order Stokes laser of KGW1 at $1493.9 \mathrm{~nm}$ is obtained. Moreover, there is no sign of crossed-cascaded SRS process, and higher order Stokes generation is cascaded SRS of each Raman crystal individually.

The total output energy and conversion efficiency of the Stokes lasers in comparison to the energy of the $1064 \mathrm{~nm}$ pumping laser is presented in Figure 3. It is most apparent that the output Stokes energy (black line) progressively increases as the energy of the pumping laser increases. However, the highest optical conversion efficiency of the SRS (red line) is $40.7 \%$ at a pumping energy of $70 \mathrm{~mJ}$. When the pumping laser reaches $120 \mathrm{~mJ}$, the total Stokes energy reaches $44 \mathrm{~mJ}$, corresponding to an optical efficiency of $36.7 \%$. 


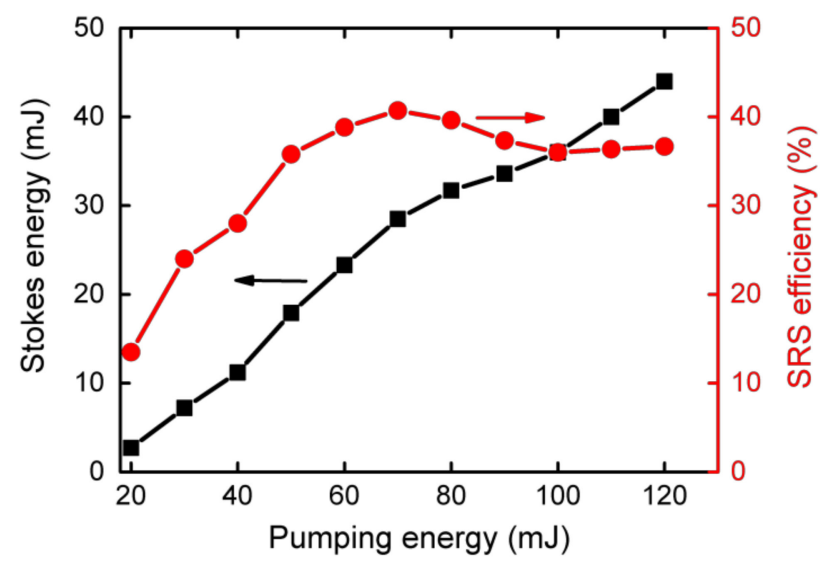

Figure 3. Output Stokes energy (black line) and optical conversion efficiency (red line) of Stokes lasers in comparison to the energy of the $1064 \mathrm{~nm}$ pumping laser.

\subsection{Multiwavelength Visible Lasers Generation}

By utilizing the Stokes lasers obtained above and keeping the pumping energy at $120 \mathrm{~mJ}$, we calculated the possible visible wavelengths from the KDP crystal and their corresponding phase-matching angles, as presented in Table 1. A total of 28 spectral lines combinations are possible after second order frequency mixing of the aforementioned seven Stokes lasers. Furthermore, 16 visible wavelengths were experimentally detected by the spectrometer (Ocean Optics HR4000 with a minimum FWHM resolution of $0.02 \mathrm{~nm}$ ), with tuning of the $\theta$-angle of the KDP crystal, also as shown in Table 1 . We can see that all angles of the experimentally detected wavelengths are from $41.8^{\circ}$ to $44.9^{\circ}$. Since some Stokes lasers are too weak to generate SHG/SFG with large signals, some red lasers cannot be detected. Moreover, some potential SHG and SFG lasers from 687.1 to $747 \mathrm{~nm}$ require too large of an incident angle for the limited aperture of the KDP crystal, and therefore have not been detected.

Considering that the KDP crystal is placed at the focus of the Stokes laser beams, laser beams in the crystal first converge and then diverge. Thus, even when the horizontal angle $\theta$ of the KDP crystal is set to a fixed value around $42^{\circ}$, the SHG and SFG processes of multiwavelength Stokes lasers can occur simultaneously. For different $\theta$ of the KDP crystal, the energies and spectra of visible lasers are shown in Figure 4. As $\theta$ increases, the spectrum of the output lasers shifts from the yellow waveband to the red waveband. Initially, when $\theta$ is $40.3^{\circ}$, only three visible wavelengths can be detected with low energy. In this case, the 588.6 and $589 \mathrm{~nm}$ wavelengths are too close to distinguish in the measured spectra. As the angle of KDP crystal increases, more distinct wavelengths appear with increased output energy. Once $\theta$ is greater than $42^{\circ}$, the red waveband becomes detectable. As $\theta$ is further increased to $43.5^{\circ}, 11$ distinct visible lasers can be obtained simultaneously. Moreover, once $\theta$ angle is increased to $45.8^{\circ}$, the longest visual wavelength of $658.48 \mathrm{~nm}$ is detected. All 16 detected visible wavelengths from 579.48 to $658.48 \mathrm{~nm}$ are presented in Figure 4a. Determined by the slit size and the grating type, the optical resolution of HR4000 spectrometer we used in this study is $\approx 0.26 \mathrm{~nm}$ (FWHM). However, the $1064 \mathrm{~nm}$ seed laser is a Q-switch laser with a narrow linewidth of $\approx 0.2 \mathrm{~nm}$, which is close to resolution of the spectrometer. And the measured spectral widths of the detected 16 visible wavelengths from 579.48 to $658.48 \mathrm{~nm}$ are $\approx 0.26 \mathrm{~nm}$. In fact, some of the aforementioned 28 calculated wavelengths may have materialized, but with such low energy to report with scientific confidence. Finally, if the $1064 \mathrm{~nm}$ laser was delivered into the KDP with other Stokes lasers, a richer spectral band could be achieved. The relationship between the total output energy of all the resulting visible lasers and the rotating angle of KDP is shown in Figure 5. It shows that maximum output energy of $8.87 \mathrm{~mJ}$ is achieved when $\theta$ is tuned at $42^{\circ}$, which corresponds to an optical conversion efficiency of $20.1 \%$. In addition, the total optical efficiency from the $1064 \mathrm{~nm}$ to visible lasers is approximately $7.4 \%$. Furthermore, when $\theta$ is larger than $42^{\circ}$, the total output energy decreases, not only due to phase 
mismatch of some output visible lasers, but also because the fundamental lasers for SHG and SFG processes are high-order Stokes lasers with lower energy.

Compared to some of the commercial wavelength tunable laser products which are based on SHG and OPO techniques, such as PHOCUS (OPOTEK, 690-950 nm), FPPO (MICROTECH, 740-1000 nm), and PhotoSonus X (EKSPLA, 665-1064 nm), this discretely tunable multiwavelength visible laser system adopts a new technique and can generate shorter tunable wavelengths varying from 579.5 to $658.4 \mathrm{~nm}$ with the same $1064 \mathrm{~nm}$ pumping laser and SHG part. Furthermore, the conversion efficiency of SRS and SHG/SFG could be improved by optimizing of time-domain waveform, beam quality, and oscillator structure in the future work. Additionally, we will strive to increase the output energy of visible-wavelength lasers for wide applications, such as spectroscopy, diagnostics, photoacoustics, multiphoton imaging, and medical research.

Table 1. Calculated and measured output wavelengths with different phase-matching angles.

\begin{tabular}{|c|c|c|c|c|c|}
\hline$\lambda_{1}(\mathrm{~nm})$ & $\lambda_{2}(\mathrm{~nm})$ & Nonlinear Process & $\lambda_{\text {cal }}(\mathrm{nm})$ & $\lambda_{\exp }(\mathrm{nm})$ & $\theta\left({ }^{\circ}\right)$ \\
\hline 1177.1 & 1177.1 & SHG & 588.6 & 588.62 & 42.0 \\
\hline 1177.1 & 1158.9 & SFG & 584 & 583.89 & 41.9 \\
\hline 1177.1 & 1197.7 & SFG & 593.7 & 593.74 & 42.2 \\
\hline 1177.1 & 1272.1 & SFG & 611.4 & 611.59 & 42.8 \\
\hline 1177.1 & 1316.7 & SFG & 621.5 & 621.92 & 43.2 \\
\hline 1177.1 & 1369.5 & SFG & 633 & 633.01 & 43.7 \\
\hline 1177.1 & 1493.9 & SFG & 658.4 & Not Detected & 45.1 \\
\hline 1158.9 & 1158.9 & SHG & 579.5 & 579.48 & 41.8 \\
\hline 1158.9 & 1197.7 & SFG & 589 & 589.07 & 42.1 \\
\hline 1158.9 & 1272.1 & SFG & 606.4 & 606.16 & 42.6 \\
\hline 1158.9 & 1316.7 & SFG & 616.4 & 616.5 & 43 \\
\hline 1158.9 & 1369.5 & SFG & 627.7 & 627.59 & 43.5 \\
\hline 1158.9 & 1493.9 & SFG & 652.6 & 652.57 & 44.9 \\
\hline 1197.7 & 1197.7 & SHG & 598.9 & 598.74 & 42.3 \\
\hline 1197.7 & 1272.1 & SFG & 616.9 & Not Detected & 43 \\
\hline 1197.7 & 1316.7 & SFG & 627.2 & Not Detected & 43.4 \\
\hline 1197.7 & 1369.5 & SFG & 638.9 & 638.93 & 43.9 \\
\hline 1197.7 & 1493.9 & SFG & 664.8 & Not Detected & 45.4 \\
\hline 1272.1 & 1272.1 & SHG & 636.1 & Not Detected & 43.7 \\
\hline 1272.1 & 1316.7 & SFG & 647 & 646.97 & 44.2 \\
\hline 1272.1 & 1369.5 & SFG & 659.5 & Not Detected & 44.8 \\
\hline 1272.1 & 1493.9 & SFG & 687.1 & Not Detected & 46.5 \\
\hline 1316.7 & 1316.7 & SFG & 658.4 & 658.48 & 44.7 \\
\hline 1316.7 & 1369.5 & SFG & 671.3 & Not Detected & 45.4 \\
\hline 1316.7 & 1493.9 & SFG & 699.9 & Not Detected & 47.2 \\
\hline 1369.5 & 1369.5 & SHG & 684.8 & Not Detected & 46.1 \\
\hline 1369.5 & 1493.9 & SFG & 714.5 & Not Detected & 48 \\
\hline 1493.9 & 1493.9 & SHG & 747 & Not Detected & 50.3 \\
\hline
\end{tabular}




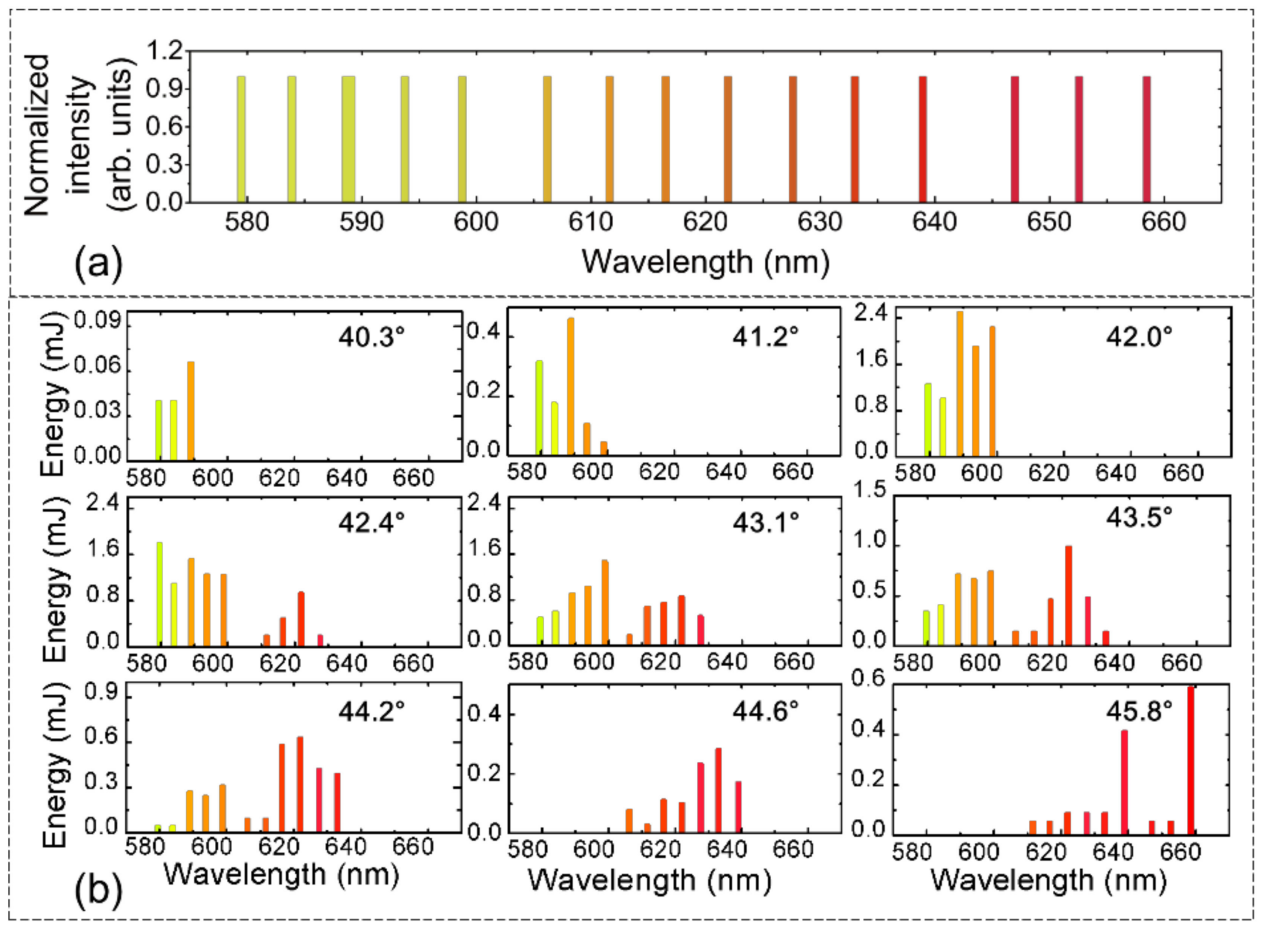

Figure 4. (a) All detected 16 visible wavelengths from 579.48 to $658.48 \mathrm{~nm}$; (b) measured spectra and energy of output visible lasers at different angles of the KDP crystal.

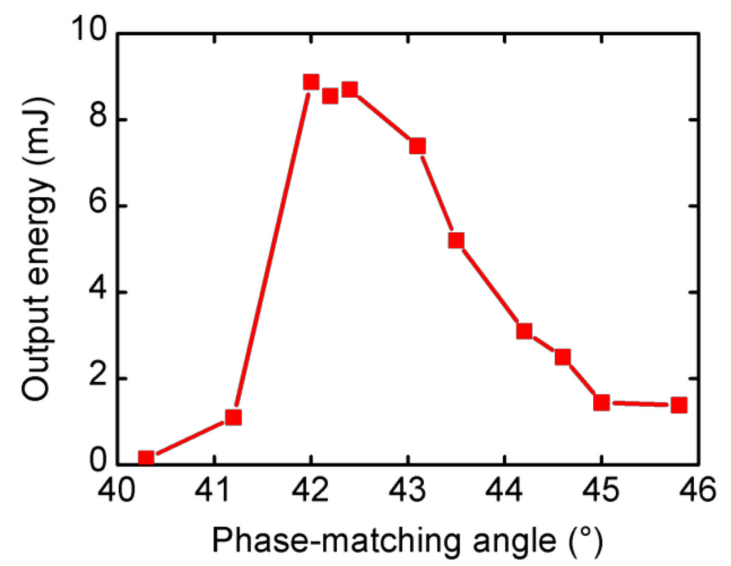

Figure 5. The energy of the visible lasers with different angles of the KDP crystal.

\section{Conclusions}

In conclusion, discretely tunable multiwavelength visible lasers were demonstrated through the solid-state SRS technique, SHG and SFG processes. The 1st, 2nd, and even 3rd-order Stokes lasers with seven wavelengths of 1158.9, 1177.1, 1197.7, 1272.1, 1316.7, 1369.5, and $1493.9 \mathrm{~nm}$ were acquired through an external Raman oscillator, which cascades one $\mathrm{Ba}\left(\mathrm{NO}_{3}\right)_{2}$ crystal with two axis-orthogonal KGW Raman crystals as the Raman gain medium. As $120 \mathrm{~mJ}$ of the $1064 \mathrm{~nm}$ laser was adopted, a maximum energy of multiorder Stokes lasers of $44 \mathrm{~mJ}$ was obtained. Moreover, the aforementioned Stokes lasers were injected as the fundamental lasers for SHG and SFG processes in a KDP crystal. A total of 16 wavelengths, varying from 579.5 to $658.4 \mathrm{~nm}$, can be generated with tuning the angle of the KDP crystal. As the $\theta$ angle of KDP increases, the spectra of output lasers shift from the yellow waveband to the red waveband. Furthermore, 11 of above-mentioned visible lasers can be produced simultaneously when the angle of the KDP crystal is tuned to $43.5^{\circ}$. The maximum energy of the 
visible lasers reached $8.87 \mathrm{~mJ}$, corresponding to an optical conversion efficiency of approximately $7.4 \%$. This technique of utilizing a multicrystal based SRS oscillator combined with SHG/SFG provides a novel method for the production of high-energy multiwavelength visible solid-state lasers, as useful for scientific and industrial applications.

Author Contributions: Conceptualization, X.L. and J.C.; Data curation, Y.L. (Yingbin Long); Formal analysis, X.L. and X.W.; Funding acquisition, Y.L. (Yuxin Leng); Investigation, X.L. and J.C.; Methodology, J.C.; Project administration, Y.P. and Y.L. (Yuxin Leng); Resources, J.C. and Y.L. (Yuxin Leng); Supervision, Y.P. and Y.L. (Yuxin Leng); Validation, X.L. and J.C.; Visualization, X.L. and G.L.; Writing-original draft, X.L. and J.C.; Writing-review and editing, Y.P., Z.H. and Y.L. (Yuxin Leng). All authors have read and agreed to the published version of the manuscript.

Funding: Shanghai Sailing Program, grant number 18YF1426200; National Key R\&D Program of China, grant number 2017YFE0123700; Strategic Priority Research Program of the Chinese Academy of Sciences, grant number XDB1603; National Natural Science Foundation of China, grant numbers 61925507 and 62075227; Program of Shanghai Academic/Technology Research Leader, grant number 18XD1404200; Shanghai Municipal Science and Technology Major Project, grant number 2017SHZDZX02; Science and Technology Commission of Shanghai Municipality, grant number 18511109702; Youth Innovation Promotion Association CAS, grant number 2020248.

Conflicts of Interest: The authors declare no conflict of interest.

\section{References}

1. Guo, H.-B.; Mai, X.; Qi, G.; Zhang, F.; Wu, G.-S.; Sun, F.; Pu, S. Multi-Wavelength Optical Storage of Diarylethene Pmma Film. Opt. Mater. 2003, 22, 269-274. [CrossRef]

2. Tan, S.; Narayanan, R.M. Design and Performance of A Multiwavelength Airborne Polarimetric Lidar for Vegetation Remote Sensing. Appl. Opt. 2004, 43, 2360-2368. [CrossRef] [PubMed]

3. Song, H.; Li, H.; Liu, X. Studies on Different Primaries for a Nearly-Ultimate Gamut in a Laser Display. Opt. Express 2018, 26, 23436-23448. [CrossRef] [PubMed]

4. Fu, R.; Deng, L.; Guan, Z.; Chang, S.; Tao, J.; Zheng, G.; Zheng, G. Zero-Order-Free Meta-Holograms in a Broadband Visible Range. Photon. Res. 2020, 8, 723. [CrossRef]

5. Stauffer, H.U.; Rahman, K.A.; Slipchenko, M.N.; Roy, S.; Gord, J.R.; Meyer, T.R. Interference-Free Hybrid Fs/Ps Vibrational Cars Thermometry in High-Pressure Flames. Opt. Lett. 2018, 43, 4911-4914. [CrossRef] [PubMed]

6. Su, W.-H.; Sun, D. Multispectral Imaging for Plant Food Quality Analysis and Visualization. Compr. Rev. Food Sci. Food Saf. 2018, 17, 220-239. [CrossRef]

7. Debord, B.; Maurel, M.; Gerome, F.; Vincetti, L.; Husakou, A.; Benabid, F. Strong Nonlinear Optical Effects in Micro-Confined Atmospheric Air. Photon. Res. 2019, 7, 1134-1141. [CrossRef]

8. Ardhendu, S.; Ray, A.; Mukhopadhyay, S.; Sinha, N.; Datta, P.K.; Dutta, P.K. Simultaneous Multi-Wavelength Oscillation of Nd Laser around $1.3 \mu \mathrm{m}$ : A Potential Source for Coherent Terahertz Generation. Opt. Express 2006, 14, 4721-4726.

9. Zhang, Y.; Xu, J.; Ye, J.; Song, J.; Yao, T.; Zhou, P.; Jiangming, X. Ultralow-Quantum-Defect Raman Laser Based on the Boson Peak in Phosphosilicate Fiber. Photon. Res. 2020, 8, 1155. [CrossRef]

10. Zhou, S.; Gu, P.; Li, X.; Liu, S. Continuous Wave Dual-Wavelength Nd: Yvo 4 Laser Working at 1064 and 1066 Nm. Chin. Opt. Lett. 2017, 15, 071401. [CrossRef]

11. Lu, Y.; Xia, J.; Liu, H.; Pu, X. Simultaneous Triple 914 Nm, 1084 Nm, and 1086 Nm Operation of a Diode-Pumped Nd: YVO4 Laser. J. Appl. Phys. 2014, 116, 163107.

12. Hou, Y.-E.; Fan, Y.-X.; He, J.-L.; Wang, H. High-Efficiency Continuous-Wave and Q-Switched Diode-End-Pumped Multi-Wavelength Nd: YAG Lasers. Opt. Commun. 2006, 265, 301-305. [CrossRef]

13. Saha, A.; Debnath, R.; Hada, D.S.; Beda, S.K. Simultaneous Oscillations of Twelve Wavelengths around $1.3 \mu \mathrm{m}$ in Quasi-CW Nd: YAG Laser. Opt. Laser Technol. 2017, 94, 112-118. [CrossRef]

14. Wang, Z.; Yang, F.; Xie, S.; Xu, Y.; Xu, J.; Bo, Y.; Peng, Q.; Zhang, J.; Cui, D.; Xu, Z. Multiwavelength Green-Yellow Laser Based on a Nd: YAG Laser with Nonlinear Frequency Conversion in a LBO Crystal. Appl. Opt. 2012, 51, 4196-4200. [CrossRef]

15. Liao, J.; He, J.; Liu, H.; Du, J.; Xu, F.; Wang, H.; Zhu, S.; Zhu, Y.; Ming, N. Red, Yellow, Green and Blue-Four-Color Light from a Single, Aperiodically Poled LiTaO3 Crystal. Appl. Phys. A 2004, 78, 265-267. [CrossRef] 
16. Yu, Q.; Wang, M.; Chen, W. Electro-Optically Q-Switched High-Repetition-Rate 1.73 Mm Optical Parametric Oscillator. Chin. Opt. Lett. 2015, 13, 081406.

17. Pask, H. The Design and Operation of Solid-State Raman Lasers. Prog. Quantum Electron. 2003, 27,3-56. [CrossRef]

18. Granados, E.; Pask, H.M.; Esposito, E.; McConnell, G.; Spence, D.J. Multi-Wavelength, All-Solid-State, Continuous Wave Mode Locked Picosecond Raman Laser. Opt. Express 2010, 18, 5289-5294. [CrossRef]

19. Wang, X.; Kang, W.; Song, X.; Xie, P.; Zong, N.; Tu, W. Theoretical and Experimental Research on High-Order Stimulated Raman Scattering in KGd(WO4)2. Opt. Commun. 2017, 385, 9-14. [CrossRef]

20. Mildren, R.P.; Butler, J.E.; Rabeau, J.R. CVD-Diamond External Cavity Raman Laser at 573 nm. Opt. Express 2008, 16, 18950-18955. [CrossRef]

21. Antipov, S.; Sabella, A.; Williams, R.J.; Kitzler, O.; Spence, D.J.; Mildren, R.P. 12 kW Quasi-Steady-State Diamond Raman Laser Pumped by an M2 = 15 Beam. Opt. Lett. 2019, 44, 2506-2509. [CrossRef] [PubMed]

22. Heinzig, M.; Vega, G.P.; Walbaum, T.; Schreiber, T.; Eberhardt, R.; Tünnermann, A. Diamond Raman Oscillator Operating at $1178 \mathrm{~nm}$. Opt. Lett. 2020, 45, 2898-2901. [CrossRef] [PubMed]

23. Lu, Y.; Cheng, W.; Xiong, Z.; Lu, J.; Xu, L.; Sun, G.; Zhao, Z. Efficient CW Laser at 559 nm by Intracavity Sum-Frequency Mixing in a Self-Raman Nd:YVO4 Laser under Direct $880 \mathrm{~nm}$ Diode Laser Pumping. Laser Phys. Lett. 2010, 7, 787-789. [CrossRef]

24. Duan, Y.; Sun, Y.; Zhu, H.; Mao, T.; Zhang, L.; Chen, X. YVO4 Cascaded Raman Laser for Five-Visible-Wavelength Switchable Emission. Opt. Lett. 2020, 45, 2564-2567. [CrossRef]

25. Basiev, T.T.; Sobol, A.A.; Zverev, P.G.; Osiko, V.V.; Powell, R.C. Comparative Spontaneous Raman Spectroscopy of Crystals for Raman Lasers. Appl. Opt. 1999, 38, 594-598. [CrossRef]

26. Findeisen, J.; Eichler, H.J.; Peuser, P.; Kaminskii, A.; Hulliger, J. Diode-Pumped Ba(No3) 2 and NaBrO3 Raman Lasers. Appl. Phys. A 2000, 70, 159-162. [CrossRef]

27. Murray, J.T.; Smith, D.; Stolzenberger, R.A.; Austin, W.; Powell, R.C.; Peyghambarian, N. Generation of 1.5-Mm Radiation through Intracavity Solid-State Raman Shifting in Ba (No 3) 2 Nonlinear Crystals. Opt. Lett. 1995, 20, 1017-1019. [CrossRef]

28. Grasiuk, A.Z.; Kurbasov, S.V.; Losev, L.L. Picosecond Parametric Raman Laser Based on KGd(WO4)2 Crystal. Opt. Commun. 2004, 240, 239-244. [CrossRef]

29. Lee, A.; Spence, D.J.; Piper, J.A.; Pask, H.M. A Wavelength-Versatile, Continuous-Wave, Self-Raman Solid-State Laser Operating in the Visible. Opt. Express 2010, 18, 20013-20018. [CrossRef]

30. Li, X. Multiwavelength Visible Laser Based on the Stimulated Raman Scattering Effect and Beta Barium Borate Angle Tuning. Chin. Opt. Lett. 2016, 14, 21404-21407.

31. Jakutis-Neto, J.; Lin, J.; Wetter, N.U.; Pask, H. Continuous-Wave Watt-Level Nd: YLF/KGW Raman Laser Operating at near-Ir, Yellow and Lime-Green Wavelengths. Opt. Express 2012, 20, 9841-9850. [CrossRef] [PubMed]

32. Mildren, R.P.; Convery, M.; Pask, H.M.; Piper, J.A.; McKay, T. Efficient, All-Solid-State, Raman Laser in the Yellow, Orange and Red. Opt. Express 2004, 12, 785-790. [CrossRef] [PubMed]

33. Liu, K.X.; Flood, C.J.; Walker, D.R.; Van Driel, H.M. Kerr Lens Mode Locking of a Diode-Pumped Nd: YAG Laser. Opt. Lett. 1992, 17, 1361-1363. [CrossRef] [PubMed]

34. Pask, H.; Blows, J.L.; Piper, J.; Revermann, M.; Omatsu, T. Thermal lensing in a barium nitrate raman laser. In Proceedings of the Paper presented at the Advanced Solid-State Lasers, Seattle, WA, USA, 28-31 January 2001.

35. Von Der Linde, D.; Maier, M.; Kaiser, W. Quantitative Investigations of the Stimulated Raman Effect Using Subnanosecond Light Pulses. Phys. Rev. 1969, 178, 11-17. [CrossRef]

36. Ding, S.; Zhang, X.; Wang, Q.; Su, F.; Li, S.; Fan, S.; Liu, Z.; Chang, J.; Zhang, S.; Wang, S.; et al. Theoretical and Experimental Research on the Multi-Frequency Raman Converter with KGd(WO4)2 Crystal. Opt. Express 2005, 13, 10120-10128. [CrossRef]

37. Ding, S.; Zhang, X.; Wang, Q.; Jia, P.; Zhang, C.; Liu, B. Numerical Optimization of the Extracavity Raman Laser with Barium Nitrate Crystal. Opt. Commun. 2006, 267, 480-486. [CrossRef]

38. Ding, S.; Zhang, X.; Wang, Q.; Su, F.; Li, S.; Fan, S.; Liu, Z.; Chang, J.; Zhang, S.; Wang, S.; et al. Highly Efficient Raman Frequency Converter With Strontium Tungstate Crystal. IEEE J. Quantum Electron. 2005, 42, 78-84. [CrossRef] 
39. Pask, H.; Dekker, P.; Mildren, R.; Spence, D.; Piper, J. Wavelength-Versatile Visible and UV Sources Based on Crystalline Raman Lasers. Prog. Quantum Electron. 2008, 32, 121-158. [CrossRef]

Publisher's Note: MDPI stays neutral with regard to jurisdictional claims in published maps and institutional affiliations.

(C) 2020 by the authors. Licensee MDPI, Basel, Switzerland. This article is an open access article distributed under the terms and conditions of the Creative Commons Attribution (CC BY) license (http://creativecommons.org/licenses/by/4.0/). 